\title{
You think you know the patient inside- out. But do you know the outside-in?
}

Emily South, Kate Khair

Haemophilia impacts on the person who has it as well as his close family and friends. The majority of healthcare provider focus is with the person with haemophilia and his carers during childhood, and then on the person himself as he becomes able to self-manage. There is a belief that the family and healthcare team support the patient equally. In this study, which was designed to understand the patient/ carer/healthcare provider relationship, we investigated support mechanisms from the patient's perspective, using narrative stories from those we call 'witnesses'. Carers, family and friends rarely feature in haemophilia research, yet can provide in-depth insight into the life of the person with haemophilia.

Three key areas were identified which underlie and cause tension in the world of haemophilia. These are described as 'identity and tackling the lion', 'the haemophilia team' and 'the unique perspective and influence of the 'outside-in'. Support from the 'outside-in' as well as healthcare providers is important for people with haemophilia for both physical and psychological health. Working together we can better support individuals with haemophilia as well as other members of their extended families. As haemophilia care

EMILY SOUTH

Innovation Director, The Planning Shop, London, UK. Email: emilysouth@planningshopintl.com

KATE KHAIR

Clinical Academic, The Centre for Outcome and Experience Research in Childhood health, Illness and Disability (ORCHID), Great Ormond Street Hospital for Children, London, UK

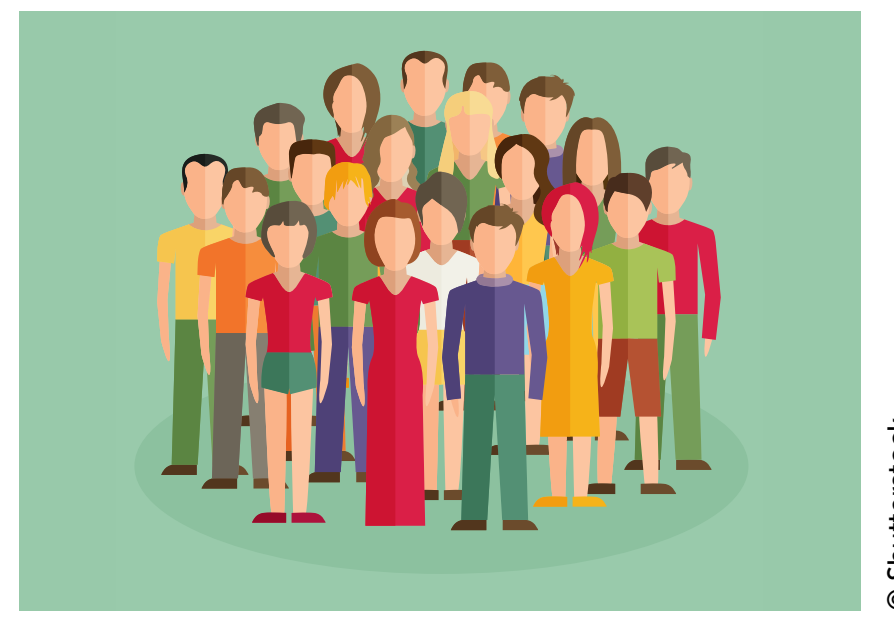

Beyond the person with haemophilia, there is a network of family, friends and partners who are also impacted. These people are 'witnesses' to the lived experience of haemophilia and, as such, may have a valuable role to play in haemophilia care

changes in a new era of therapeutic options; we need to re-evaluate the supportive role of family and carers to ensure that the patient and family voice is heard in decision-making at an individual and national level.

Keywords: haemophilia, inside-out, outside in, patient experience, self-management

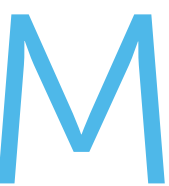

aking assumptions can be an important first step to solving problems. But it can also bias how we frame challenges, the questions we ask, and the decisions we make. It is the same for healthcare market research and pharmaceutical interactions with healthcare providers. The way we think about individuals living with illness, and how we design research and create solutions, 
can be led by our own biases and assumptions about people. Thirsk and Clark, for example, state that "The focus on 'self' in 'self-management' can lead us to think about chronic diseases as a largely independent activity and that people require individual solutions" [1].

In addition, the word 'management' implies that there is a set of strategies that everyone can work with consistently and effectively. When designing research methodologies, we tend to zoom in on the main characters to learn about their experiences: the person living with the illness, the physician, perhaps the carer or the nurse. And when understanding prescribing behaviours, we focus in on the physician-patient consultation.

But when this 'individual focus' does not tell the whole story, we miss out on a valuable detail. The missing pieces are key to the research objectives or to the care we deliver. Focusing on the individual may not be the most accurate way to tell an individual story. Indeed, chronic diseases can be viewed in a more connected sense, "embedded in family, community and societal conditions that shape and influence" [2]

Furthermore, we are all unreliable witnesses of our own behaviour. This is further complicated for people living with a long-term illness due to emotional burden, which may impact on recall and result in them sharing only what they believe healthcare professionals want to hear. This is known as biographical disruption, and was first defined by Bury to explain the disruption to an individual's self-narrative and self-concept that results from chronic illness ${ }^{[3,4]}$.

This study was undertaken by a market research company (The Planning Shop) to understand how people with haemophilia deal with the day-to-day realities of living with a long-term condition and the frequent treatment administrations necessary, as well as the impact of living with haemophilia on immediate family and friends.

Haemophilia: invisible, unpredictable, malicious Haemophilia is a rare, usually inherited, genetic disorder that inhibits the blood's ability to clot, with types A and B affecting factor VIII and factor IX respectively. Annual data from the United Kingdom Haemophilia Centres Doctors' Organisation (UKHCDO) in 2017 shows 9,639 people (mostly males) living with haemophilia, with 2,295 having severe disease ${ }^{[5]}$. These numbers exclude females who may also have symptoms and may be considered to also have haemophilia ${ }^{[6]}$, and the wider sphere of different family and friends impacted by haemophilia.
Haemophilia can be described as:

Invisible: Bleeding is the most identifiable sign, but this is often accompanied by chronic joint pain and psychosocial issues. The combined limitations and lack of wider community understanding can make haemophilia antisocial and isolating, lending a wider meaning to 'invisible'.

Unpredictable: Even the most experienced person cannot always anticipate when or how haemophilia will strike. A bleed may result from a repetitive task at work, a night out with friends, a simple knock at home, a trip on the pavement - or even occur spontaneously.

Malicious: As a haemophiliac, you may be busy with life one day and temporarily debilitated and using a wheelchair, the next. Haemophilia can interrupt important plans and negatively impact quality of life.

\section{METHODS}

The Planning Shop conducted this market research to challenge what we call an 'island view' of the patient, by introducing 'witnesses' to our studies. Witnesses are the people (carers, family members, partners, friends) who are usually absent from patient research but who have an important perspective and can potentially influence patient management and treatment of individuals with health conditions. Taking severe haemophilia as an example, we recently undertook a qualitative research project to understand whether or not witnesses could add value to patient research. Witnesses were included in the research in order to help build a more complete picture and to uncover deeper insights into living with haemophilia. By harnessing the individualised support and mentoring that witnesses provide to patients, we believe we can promote more effective patient care and engagement.

The study was conducted using an open approach that allowed for the inclusion of people in a wider range of clinical and non-clinical roles than we would usually interview in patient research. The recruitment process involved:

- Mapping all potential 'touchpoints' of a person living with haemophilia (ie people considered to be in their team)

- Using descriptive statements in the screening materials to outline the potential influence and involvement of 'witnesses' as well as the 'main character' roles

- The inclusion of a diverse sample of individuals who view the experience of haemophilia from different angles. 
Our methodology included the use of indirect questioning, self-discovery exercises and challenging team members with different viewpoints. We explored the most important connections and relationships with healthcare professionals, people with haemophilia and their family members through both remote discussions using an online platform and face-to-face interviews, to build depth and breadth of understanding of the study participants and their supporters.

\section{RESULTS}

An underlying tension in the world of haemophilia Advances in treatment have gradually moved haemophilia care from the hospital into the community. Comprehensive Care Centres (CCCs) with in-house multidisciplinary clinical teams - including the haematologist, specialist nurse and physiotherapist (and psychologist in larger centres) - are now providing hubs of expertise designing treatment and management plans, where people living with haemophilia can selfmanage in the community and live their lives ${ }^{[7,8]}$.

However, our research uncovered an underlying tension in haemophilia: living an independent life and self-growth in a medicalised system. This tension results in an ongoing risk-benefit gamble for patients and carers throughout their lives, with haemophiliacs asking themselves questions like:

- "Is it worth the risk of a bleed or chronic joint and pain issues?"

- "Shall I follow the rules?"

- "Who am I?"

Our research has shed light on how people living with haemophilia find their way around this tension in three 'insights':

Insight 1: Identity and 'tackling the lion'

To begin to explain this tension, a story about Hercules provides a useful analogy.

In Greek mythology, the first of Hercules' 12 tasks was to kill the vicious Nemean lion. After much persistence, Hercules killed the lion with the force of his hands. Once dead, he removed the lion's skin and used it like armour, making him invincible. In psychological terms, to fight the lion means to face our deepest fears, but to accept our weaknesses as part of our identity (wearing the skin) is to be transformed.

Interestingly, the goal of being brave and wearing your haemophilia with pride is what the healthcare professionals (HCPs) we spoke with try to inspire in people living with haemophilia. Influential role models wear their haemophilia in a similar way: for example, Christian L. Harris, whose severe haemophilia A has inspired his fashion designs ${ }^{[9]}$, or Chris Bombardier, who is raising awareness by completing the Seven Summits mountaineering challenge with severe haemophilia $\mathrm{B}^{[10]}$. However, this is not a realistic and desirable goal for all people with severe haemophilia.

We have identified different and overlapping paths taken by people living with haemophilia in our study:

- The 'regular guy': Through adjustments and adaptions, haemophilia is kept in the background and does not play a starring role in the individual's life. A sense of wellbeing and belonging is achieved.

- Social withdrawal: Patients can be derailed by the limitations placed on them by their haemophilia, leading to social withdrawal and isolation

- Low self-worth: The individual's personal identity can be consumed by the negative associations of haemophilia and they can experience a loss of self-worth.

Those affected by the contaminated blood scandal of the 1970s and 1980s that live with blood-borne viral illnesses may be particularly prone to the 'cul-de-sacs' of low self-worth and/or social withdrawal due to limitations posed by their fluctuating clinical condition. The paths for someone living with haemophilia are not consistent or straightforward, which lends value to understanding the perspective of the 'witnesses' who share their experience.

\section{Insight 2: The haemophilia team}

The people with haemophilia in our research acknowledged that a team of individuals surrounds them. One person said that he and his team collectively form 'one functional person'. When looking at the carer parent (typically mothers) and paediatric team versus the adult team, we noticed they are reportedly different in size: parent and paediatric teams tend to be large and varied, while adults with haemophilia report having a smaller, more intimate team. During clinical transition (from paediatric to adult care) teens and young adults described leaving behind what was their parents' team and building their own trusted team from scratch. This was most apparent for those who moved geographically from a paediatric centre to an adult haemophilia centre.

Partners, spouses or best friends were often reported as the adult patient's most influential team members - more so than the medical team. The 'patient team' evolves over time through the course of different life events (eg relocating, marriage, having 
children, etc). A partner or best friend (unlike the medical team) can be confided in over the long term, offering regular emotional and practical support.

Conversely, our research showed that the clinical teams perceived things differently: they acknowledged that the person living with haemophilia has a team surrounding them, but over-estimated the influence of wider friends, immediate family and independent organisations or charities rather than the immediate trusted team. How does this happen? People living with haemophilia stated that wider friends and immediate family "don't get it", that patient organisations do not offer valuable support locally, or that they are more comfortable with others not knowing too much about their haemophilia because it creates too many questions and distractions in their everyday lives. There may also be geographical, cultural and religious reasons for people not being open about their health status ${ }^{[11]}$.

Importantly, the clinical team over-estimated their own influence, believing they offered more emotional support than people with haemophilia thought they did - sometimes this 'emotional support' was offered under the guise of education.

We demonstrated three core reasons for these disconnects and tensions:

1. Geography and contact: The geographical distance between CCCs and people living with haemophilia and their families, with only once- or twice-yearly face-to-face interactions with the clinical team

2. The physician focus on clinical aspects of haemophilia: 'Blood and joints' and the assumption that haemophilia is 'manageable' for everybody

3. Simultaneous trust and distrust: Trust in medical expertise, but lack of trust in the medical team's understanding of the individual's life and desires.

The reality of living with haemophilia is complex. Patients surround themselves with a trusted network of individuals. Speaking with these 'team' members is vital to understanding how people navigate their world with haemophilia; however, the clinical care team perspective does not appear to understand this fully. The most critical team members are a subset of the witnesses we spoke to and represent a new target for research we have called these the 'outside-in'.

Insight 3: The unique perspective and influence of the 'outside-in'

The 'outside-in' team members of the person living with haemophilia or parent carer:

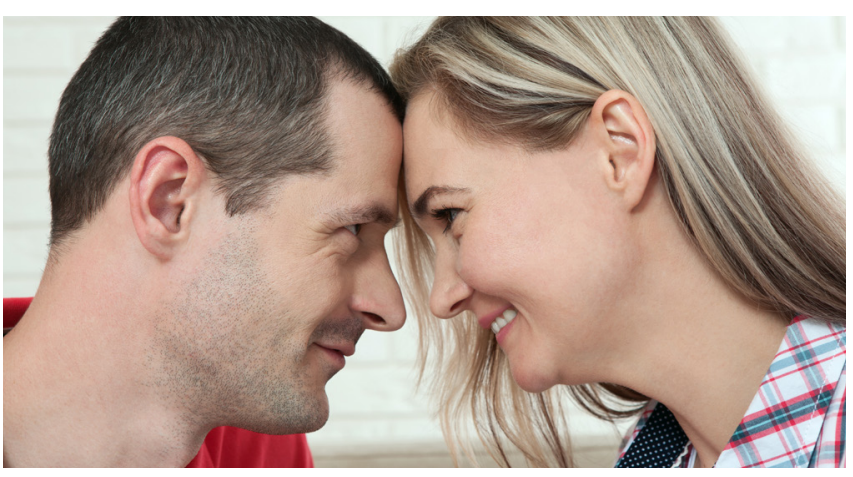

Members of the 'outside-in' know the individual living with haemophilia well, play an influential role in their lives, and can help to create a more complete picture of their lived experience. Their unique perspective and involvement in the wider care team can help in providing better support

- Are not necessarily part of the multidisciplinary clinical team or part of their family or friends

- Have come from the outside and are now on the inside, meaning they are able to give a fresh perspective on haemophilia (eg a new romantic partner)

- Know the person living with haemophilia or parental carer inside-out.

In our research we identified many examples of 'outside-in' team members. These individuals are in a unique position to not only share their view of the person living with haemophilia's world and experience, but also to take an influential role and effect change.

An example of an 'outside-in' team member who took part in our research is Sarah (names changed in subsequent text to maintain anonymity). Sarah is the relatively new partner of John, who lives with severe haemophilia. The couple shared some interesting anecdotes during a face-to-face interview.

Sarah activates and augments John's engagement with treatment. In discussions, John compared his adherence to treatment before and after they met:

"I know sometimes they [the bleeds] go untreated - I would say that's quite rare now... $10 \%$ would get treated previously, and 10\% might be left untreated this time round."

Sarah takes an ongoing role in monitoring and communicating John's treatment via the Haemtrack app. John takes a more passive role and said:

"I wouldn't do that. I'd forget to be quite honest. So, you've [Sarah] got it on your phone. I haven't got it on my phone." 
Sarah also helps to filter information to John in a way she knows is digestible, from online sources and by being an active member of the Haemophilia Society (unlike John).

Haemophilia care is not just about looking after the immediate patient; it is about future generations too. During the interviews, Sarah described how she "dropped the word 'daughters' into a conversation with John's long-term haematologist" at a routine clinic review. She reported that she did this intentionally with the aim of creating a 'domino effect': Sarah recognised that John's daughters were obligate carriers of haemophilia, as did John and the haematologist, but there had been no proactive conversations about it. John said he believed:

"It would have been on the [medical] record, but then it just gets lost."

Sarah clarified that a conversation with the haematologist would never have been prompted by John or the clinician without her, and said:

"I know what questions to ask... maybe nobody else would."

As a direct result of Sarah's intervention, John's daughters are now receiving adequate information on haemophilia and available treatments, and can use this information when they start to plan their own families.

Sarah's anecdotes revealed that the 'outside-in' are highly conscious of the person with haemophilia's experience and are influential within their world. Our research identified many other individuals like this, both clinical and non-clinical: for example, a support worker, who had a unique bird's eye view of the multidisciplinary team, provided a view from people who would never usually take part in research, reporting a young man with social and housing issues who had retreated to online gaming to actively avoid managing his haemophilia.

In summary the 'outside-in':

- Create positive adjustments and adaptations to the person with haemophilia's lifestyle

- Are highly engaged and influential in haemophilia treatment and management

- Enable the people they care about to live in the moment, rather than reflect negatively on the past or worry unduly about the future.

\section{DISCUSSION}

Ultimately the 'outside-in' are creating resilience and ongoing functional wellbeing - more complex than simply emotional or practical support. As time moves on they expect to have an increased role in the lives of the people with haemophilia they support.

We identified individuals from the study cohort who were significant influencers and who helped us to piece together a more accurate picture of the patients' experiences. It was also valuable to speak with other 'team' members, especially in collaboration with the person living with haemophilia. For example, where the individual with haemophilia was unable to express himself the team member was able to fill in the gaps. Their presence in the conversation and acknowledgement of the issues led to more natural and collaborative discussions

Importantly, by speaking with the 'outsidein' and other team members, we were able to gain a more complete picture of the individual's experiences with haemophilia and understand how the important tensions in haemophilia referred to earlier are navigated. This provides a solid platform for communication between the patient, his family and the multidisciplinary care team (MDT), which will enable solution generation if potential conflicts, perhaps around treatment options or adherence, occur.

Our findings raise important considerations for the clinical approach to haemophilia care in the UK National Health Service (NHS). The focus on independence and self-management does not necessarily reflect the reality of what management in the community really looks like. There is potentially a need to rethink how healthcare professionals support and engage in the individual's world via the patient's personal team (as far as privacy considerations allow). Adults who wish to bring their personal team into their relationship with the clinical team, for example by attending clinic visits, should be encouraged to do so: the involvement can add valuable insights that impact personalised treatment and management decisions.

Anecdotally, mothers of adults with haemophilia who still attend clinic appointments with their sons are often considered to be 'over-protective', unable to 'let go of their child', and may even be labelled as feeling 'guilty' for passing on haemophilia to their sons. Beeton et al. reported the deep emotional impact of having a child with haemophilia and that this had a transformational impact on the whole family ${ }^{[12]}$. Of course, mothers of men with haemophilia are often also carriers of haemophilia themselves, even if not 
clinically affected ${ }^{[6]}$. These women have been shown to have lower health-related quality of life when compared to mothers of children with other longterm conditions ${ }^{[13]}$, and may gain personal support and knowledge when attending these clinics. Doltani et al. have shown a positive influence on doctor/patient relationships when patients are accompanied in the clinic, with the accompanying person (usually a parent or spouse) being advocates for the patient, which is reported to enhance doctor-patient communication ${ }^{[14]}$

The MDT should engage with the family team to establish the views and reasons for all family team members attending clinic. Is it that the carers or female family members are seeking information about the person with haemophilia or for themselves about their own bleeding problems? Khair et al. demonstrated that even in large haemophilia centres there is a substantial subgroup of young women who experience stigmatisation, isolation and bullying, and express concerns relating to fertility, conception and future family planning that are not routinely addressed ${ }^{[15]}$. This reflects the example of John's daughters who, as haemophilia carriers, were not recognised as patients themselves.

Haemophilia treatment is currently facing a 'tipping point' with new and innovative treatments, with disruptive therapies and gene therapy becoming realistic treatment options for many. Before embarking on these new therapies, careful consideration is needed by the patient and his family. The new therapies may result in less need for hospital-delivered care, which may lead to more isolation for some people with haemophilia. There is concern that the daughters of these men, who have even less contact with haemophilia centres, will be more 'lost' in future haemophilia care delivery. If their fathers are seen in clinic less often, will these girls become invisible to healthcare providers and suffer the consequences of this later in life? ${ }^{[16]}$

\section{The patient's world}

Haemophilia treatment has come a long way since the early days of patriarchal treatment where the doctor knew best. The MDT now acknowledges patients as engaged and influential people in treatment and self-management. It is now time to zoom out to reveal the world of the person - a wider web of social interactions and situations - a more complex system of decision influences and triggers. We believe this broader picture is currently an untapped resource of experiences and insights across haemophilia care. Involving patients and family members/caregivers in goal-setting, using methods such as motivational interviewing, help to support treatment concordance, resulting in better outcomes that are relevant to people with haemophilia ${ }^{[17,18]}$. Individualising clinical reviews, including the possibility of telephone follow-up and support for those treated with contemporary and new therapies, should be considered to limit disruption to busy patient and family lives. Telephone follow-up is a method of review that is already used and popular with patients, promoting engagement with the haemophilia centre and ensuring continued education ${ }^{[19]}$.

\section{Solutions driven by the 'outside-in'}

Our research has shown that the 'outside-in' team can have a transformational effect and can be important influencers in the world of the person with haemophilia. Cassis et al. report how relationships are affected by haemophilia in various ways, and particularly with regard to disease burden, age and social life ${ }^{[20]}$. We suggest utilising the care of the 'outside-in' to offer better support those with haemophilia ${ }^{[21,22]}$. As experts in the desires and the day-to-day quality of life of people who have haemophilia, and with experience in creating lifestyle adjustments, insights from the 'outside-in' can potentially help to develop solutions that connect healthcare teams and patients better, are more sustainable and work beyond an individual level - perhaps through local and national haemophilia patient support groups.

\section{Diverse engagement}

People with haemophilia and their families need to be able to engage with multiple team stakeholders, adapting across life stages with support from healthcare providers that is tailored to the right people at the right time (eg at transition from paediatric to adult care, when undergoing surgery, with ageing). As services and new therapies evolve, healthcare professionals need to be conscious of 'connectability' and input from members of the wider team, outside of the immediate comprehensive care team, to ensure optimal communication with patients and their families.

\section{Communications through the 'lens of life'}

Healthcare professionals should create new models of clinically-focused care in order to improve engagement with patients and carers who are living with haemophilia Ultimately, building strong relationships and trust between the patient, his family and healthcare providers will help the clinical team to quickly understand the patient/family context and desires in consultations, to create tailored solutions through tools such as motivational interviewing and goal-setting ${ }^{[16,17]}$. This can 
also be used to inform the creation of resources that move beyond educating about medical moments such as treatment episodes and clinic visits to help people live demonstrably better lives, including reduced bleeds, and better joint health and quality of life.

\section{CONCLUSION}

As we enter a new era of haemophilia care, with nonfactor replacement and gene therapy on the horizon, we need to understand the world and experiences of people with haemophilia as clearly as we can, in order to maintain a cohesive, engaged team, with all participants working towards the best outcomes for patients and families. Healthcare professionals need to understand all of the factors that influence patients and families, as these will impact on future engagement within clinical care and follow-up. The 'outside-in' can be used to create new and valuable care models that speak to and for the people that use them.

\section{ACKNOWLEDGEMENTS}

A version of this manuscript was presented to the British Healthcare Business Intelligence Association and is available at https://pharmaphorum.com/ patients/insights-patients/how-pharma-can-boostengagement-by-embracing-support-networks/

Informed consent has been obtained from the participants in the study reported in this paper.

\section{REFERENCES}

1. Thirsk LM, Clark AM. What is the 'self' in chronic disease selfmanagement? Int Journal Nurse Studies 2014; 51(5): 691-3.

2. Kendall P. Investing in prevention: improving health and creating sustainability. The Provincial Health Officer's special report. British Columbia: Office of the Provincial Health Officer, 2010. Available from https://www.health.gov.bc.ca/ library/publications/year/2010/Investing_in_prevention_ improving_health_and_creating_sustainability.pdf (accessed 16 November 2018).

3. Bury M. The sociology of chronic illness: a review of research and prospects. Sociol Health Illn 1991; 13(4): 451-68.

4. Bury M. Chronic illness as biographical disruption. Sociol Health Illn 1982; 4(2): 167-82.

5. UKHCDO. UK bleeding disorder statistics for April 2016 to March 2017. A report from the UK National Haemophilia Database. 2017. Available from http://www.ukhcdo.org/wpcontent/uploads/2018/02/Bleeding-Disorder-Statistics-forApril-2016-to-March-2017-for-UKHCDO.pdf (accessed 16 November 2018).

6. Sager R. Women with haemophilia: more than just carriers. J Haem Pract 2014; 1(2): 2-7. doi: 10.17225/jhp.00016.

7. Khair K, Meerabeau L, Gibson F. Self-management and skills acquisition in boys with haemophilia. Health Expect 2015; 18(5): 1105-13.
8. Schrijvers LH, Schuurmans MJ, Fischer K. Promoting selfmanagement and adherence during prophylaxis: evidencebased recommendations for haemophilia professionals. Haemophilia 2016; 22(4): 499-506.

9. Marshall B. Fashion statement: hemophilia-inspired clothing line garners national attention. HemAware 17 November 2014. Available from https://hemaware.org/community-pulse/ fashion-statement (accessed 16 November 2018).

10. Henriques $\mathrm{C}$. Chris Bombardier becomes first hemophiliac to climb 7 continents' high peaks. Hemophilia News Today 7 February 2018. Available from https://hemophilianewstoday. com/2018/02/07/hemophiliac-chris-bombardier-becomesfirst-ever-to-climb-seven-summits/ (accessed 16 November 2018).

11. Goldstein $G$, Kenet $G$. The impact of chronic disease on the family. Haemophilia 2002; 8(3): 461-5.

12. Beeton $K$, Neal D, Watson T, Lee CA. Parents of children with haemophilia - a transforming experience. Haemophilia 2007; 13(5): 570-9.

13. Gilbert L, Paroskie A, Gailani D, Debaun MR, Sidonio RF. Haemophilia A carriers experience reduced health-related quality of life. Haemophilia 2015; 21(6): 761-5.

14. Doltani D, Imran A, Saunders J, Harmon D. Who accompanies patients to the chronic pain clinic? Ir J Med Sci 2017;186(1): 235-8.

15. Khair K, Holland M, Pollard D. The experience of girls and young women with inherited bleeding disorders. Haemophilia 2013; 19(5): e276-81.

16. von der Lippe C, Frich JC, Harris A, Solbraekke KN. "It was a lot tougher than I thought It would be". A qualitative study on the changing nature of being a hemophilia carrier. J Genet Couns 2017; 26(6): 1324-32.

17. Roberts JC, Lattimore S, Recht M, et al. Goal attainment scaling for haemophilia (GAS-Hēm): testing the feasibility of a new patient-centric outcome measure in people with haemophilia. Haemophilia 2018; 24(4): e199-e206.

18. Gue D, Squire $S$, Mclntosh $K$, et al. Joining the patient on the path to customized prophylaxis: one hemophilia team explores the tools of engagement. J Multidiscip Healthc 2015; 7(8) :527-34.

19. Alavian S, Hutchinson W, Morris A, Williams H, Pollard D. Nurse-led telephone review service for mild inherited bleeding disorders improves attendance rates, frees hospital resources and is highly rated by patients. J Haem Pract 2018; 5(1): 66-75. doi: 10.17225/jhp00113.

20. Cassis FR, Buzzi A, Forsyth A, et al. Haemophilia Experiences, Results and Opportunities (HERO) Study: influence of haemophilia on interpersonal relationships as reported by adults with haemophilia and parents of children with haemophilia. Haemophilia 2014;20(4): e287-95.

21. Khair K, Gibson F, Meerabeau L. 'I can always rely on them': the importance of social support for boys with haemophilia. $J$ Haem Pract 2014; 1(1): 10-16.

22. Torres-Ortuño A, Cid-Sabatel R, Barebero J, Garcia-Dasi $M$. Life experience of the adult and ageing patient with haemophilia. Practical aspects for psychological support. Vox Sang 2017; 112(4): 301-9. 


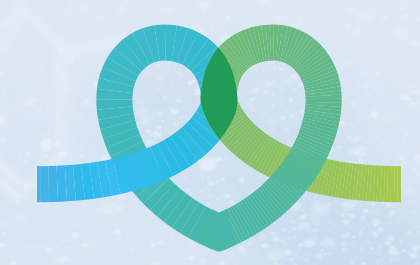

\section{The Journal of Haemophilia Practice}

An open-access journal for sharing experience in the care of people with bleeding disorders 